\title{
Novel Full-Diversity High-Rate STBC for 2 and 4 Transmit Antennas
}

\author{
Sushanta Das, Naofal Al-Dhahir, and Robert Calderbank
}

\begin{abstract}
We design a new rate- $\frac{5}{4}$ full-diversity orthogonal space-time block code (STBC) for QPSK and 2 transmit antennas (TX) by enlarging the signalling set from the set of quaternions used in the Alamouti [1] code. Selective power scaling of information symbols is used to guarantee full-diversity while maximizing the coding gain (CG) and minimizing the transmitted signal peak-to-minimum power ratio (PMPR). The optimum power scaling factor is derived analytically and shown to outperform schemes based only on constellation rotation while still enjoying a low-complexity maximum likelihood (ML) decoding algorithm. Finally, we extend our designs to the case of 4 TX by enlarging the set of Quasi-Orthogonal STBC with power scaling. Extensions to general M-PSK constellations are straightforward.
\end{abstract}

Index Terms-STBC, quaternions, coding gain, peak-tominimum power ratio.

\section{INTRODUCTION}

$\mathbf{O}$ UR objective in this letter is to design a new class of fulldiversity high-rate $(>1)$ space-time block codes (STBC) by exploiting the inherent algebraic structure in existing orthogonal designs based on quaternions for 2 transmit antennas [1] and quasi-orthogonal designs for 4 transmit antennas [5]. The simplest example of a complex orthogonal design is the $2 \times 2$ code

$$
\mathcal{Q}\left(x_{1}, x_{2}\right) \rightarrow\left[\begin{array}{cc}
x_{1} & x_{2} \\
-x_{2}^{*} & x_{1}^{*}
\end{array}\right]
$$

discovered by Alamouti [1] where (.)* denotes the complexconjugate transpose. This code achieves rate- 1 at full diversity and enjoys low-complexity ML decoding using matched filtering. The correspondence between Alamouti matrices and quaternions means that the set of Alamouti matrices is closed under addition, multiplication and inversion. Consider the set $\tilde{\mathcal{Q}}$ of $\mathbf{x}$ given by $2 \times 2$ orthogonal matrices

$$
\tilde{\mathcal{Q}}\left(x_{1}, x_{2}\right)=\left[\begin{array}{cc}
x_{1} & x_{2} \\
x_{2}^{*} & -x_{1}^{*}
\end{array}\right]=\left[\begin{array}{cc}
1 & 0 \\
0 & -1
\end{array}\right] \mathcal{Q}\left(x_{1}, x_{2}\right)
$$

Then, $\mathcal{Q}$ is a multiplicative group, $\tilde{\mathcal{Q}}$ is a coset of $\mathcal{Q}$, and the union $\mathcal{S}=\mathcal{Q} \cup \tilde{\mathcal{Q}}$ is also a multiplicative group. For $\mathbf{E}, \mathbf{F} \in$

Manuscript received September 14, 2005. The associate editor coordinating the review of this letter and approving it for publication was Prof. GiHong Im. The work of N. Al-Dhahir is supported in part by the Texas Advanced Technology (ATP) program project no. 009741-0023-2003, and by NSF contracts no. CCF 0430654 and DMS 0528010.

S. Das is with Philips Research North America, Briarcliff Manor, NY (email: sushanta.das@philips.com).

N. Al-Dhahir is with the University of Texas at Dallas, Richardson, TX (e-mail: aldhahir@utdallas.edu).

R. Calderbank is with Princeton University, Princeton, NJ (e-mail: calderbank@math.princeton.edu).

Digital Object Identifier 10.1109/LCOMM.2006.03032.

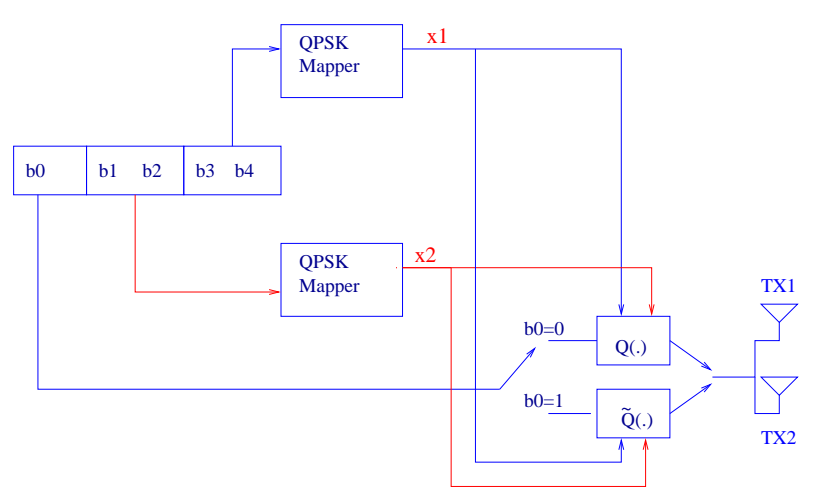

Fig. 1. The block diagram of rate- $\frac{5}{4}$ STBC for QPSK modulation.

$\tilde{\mathcal{Q}} \rightarrow \mathbf{E F} \in \mathcal{Q}$ while for $\mathbf{E} \in \mathcal{Q}$ and $\mathbf{F} \in \tilde{\mathcal{Q}} \rightarrow \mathbf{E F} \in \tilde{\mathcal{Q}}$. We will use the expanded set $\mathcal{S}$ to construct new high-rate $(>1)$ fulldiversity space-time block code with low complexity decoding and optimized available coding gain.

\section{Proposed CODE FOR 2 TX}

\section{A. Transmission Scheme}

The columns of $\mathcal{Q}$ represent different antennas, the rows represent different time slots, and the entries are the two symbols to be transmitted assuming a quasi-static flat-fading channel. Our code construction is applicable to any M-PSK constellation. However, to simplify the presentation, we will focus on $Q P S K$ modulation. In our proposed scheme, the transmitted space-time signaling matrix is selected from either $\mathcal{Q}$ or $\tilde{\mathcal{Q}}$ according to an additional information bit of 0 or 1, respectively (Fig.1). Hence, the proposed scheme achieves a $25 \%$ information rate increase compared to the traditional Alamouti scheme for $Q P S K$ modulation without requiring any additional system resources (power or bandwidth).

\section{B. Code Design Criteria}

Consider two distinct codewords $\mathbf{S}_{i}, \mathbf{S}_{j} \in \mathcal{S}$. In order to ensure full spatial diversity, the codeword difference matrix $\mathbf{B}=\left(\mathbf{S}_{i}-\mathbf{S}_{j}\right)$ between any two distinct codewords in the extended set $\mathcal{S}$ must have full rank [4]. When both codewords $\mathbf{S}_{i}$ and $\mathbf{S}_{j}$ belong to $\mathcal{Q}$ or $\tilde{\mathcal{Q}}$, $\mathbf{B}$ will be full rank. However if $\mathbf{S}_{i} \in \mathcal{Q}$ and $\mathbf{S}_{j} \in \tilde{\mathcal{Q}}$ (or vice versa), B loses rank. To restore full-diversity, schemes based on rotations of information symbols have been proposed (see e.g. [3], [7]). In this letter, we propose to divide the information symbols in $\tilde{\mathcal{Q}}$ only by a real scalar $K(>1)$ to guarantee full-diversity, hence the name selective power scaling. For a unit-radius 
QPSK constellation, this scaling results in an overall signal constellation consisting of two concentric circles of radii 1 and $\frac{1}{K}$.

\section{Finding the Optimum Power Scaling Factor}

The main objective of introducing the power scaling factor $K$ is to ensure full diversity for the proposed high-rate STBC. Since $K>1$, the average transmitted power is even reduced compared to the case of no scaling. Two important selection criteria for $K$ are maximizing the CG and minimizing the PMPR resulting from power scaling. If the QPSK symbols on the outer constellation circle are normalized to unity, and the radius of the inner circle is $\frac{1}{K}$, the PMPR equals $K^{2}$. In addition, $\mathrm{CG}$ is defined as the minimum product of the nonzero singular values of $\mathbf{B}$ over all distinct codeword pairs. We propose to select $K$ by optimizing the cost function:

$$
\begin{aligned}
K_{\text {opt }} & =\arg \max _{K>1} \frac{C G}{P M P R} \\
& =\arg \max _{K>1} \frac{\min _{\mathbf{S}_{i} \in \mathcal{Q}, \mathbf{S}_{j} \in \tilde{\mathcal{Q}}, \mathbf{S}_{i} \neq \mathbf{S}_{j}} \operatorname{det}\left(\mathbf{B B}^{*}\right)}{K^{2}}
\end{aligned}
$$

The two codewords have the form

$$
\mathbf{S}_{i}=\left[\begin{array}{cc}
x_{1} & x_{2} \\
-x_{2}^{*} & x_{1}^{*}
\end{array}\right], \quad \mathbf{S}_{j}=\left[\begin{array}{cc}
\frac{y_{1}}{K} & \frac{y_{2}}{K} \\
\left(\frac{y_{2}}{K}\right)^{*} & -\left(\frac{y_{1}}{K}\right)^{*}
\end{array}\right]
$$

$$
\begin{aligned}
\text { Hence, } \operatorname{det}\left(\mathbf{B B}^{*}\right) & =\operatorname{det}(\mathbf{B}) \operatorname{det}\left(\mathbf{B}^{*}\right) \\
& =\frac{\left[\left(1-K^{2}\right)^{2}+K^{2}\left[\Im\left(x_{1} y_{1}^{*}+x_{2} y_{2}^{*}\right)\right]^{2}\right]}{K^{4}}
\end{aligned}
$$

There exists specific choices of $\{\mathbf{x}\}$ and $\{\mathbf{y}\}$ (e.g. when $x_{1}=$ $y_{1}$ and $x_{2}=-y_{2}$ ) that result in $x_{1} y_{1}^{*}+x_{2} y_{2}^{*}=0$ and these choices set the minimum value of $\operatorname{det}\left(\mathbf{B B}^{*}\right)$ with respect to $\{\mathbf{x}\}$ and $\{\mathbf{y}\}$ and irrespective of $K$. Therefore, we can write

$$
K_{\text {opt }}=\arg \max _{K>1} \frac{\left[1-K^{2}\right]}{K^{3}}
$$

Simple first and second derivative tests yield that $K_{\text {opt }}=\sqrt{3}$. Similarly, for any M-PSK constellation, the value of $K$ can be optimized offline as a function of $M$ and the achievable rate in this case is $1+\frac{1}{2 \log _{2} M}$.

\section{Low-Complexity Decoding}

The output symbols $r_{1}, r_{2}$ received over two consecutive symbol periods can be represented as follows:

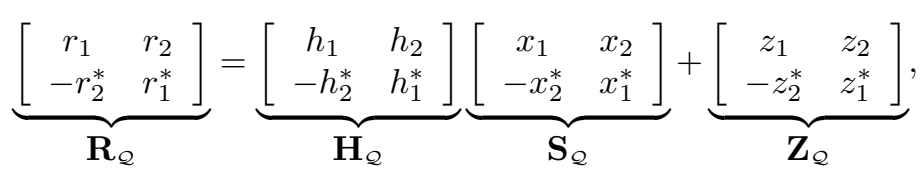

and

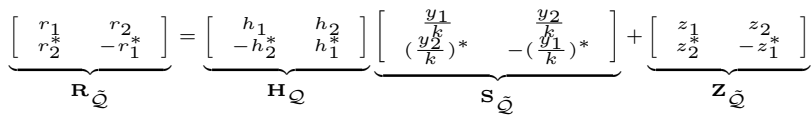

where $\mathbf{R}_{\mathcal{Q}}$ and $\mathbf{R}_{\tilde{\mathcal{Q}}}$ are $2 \times 2$ complex matrix representations of the received signals corresponding to transmitted codewords in the form of $\mathcal{Q}$ and $\tilde{\mathcal{Q}}$, respectively. The path gains from the two transmit antennas to the mobile are $h_{1}, h_{2}$, and the noise samples $z_{1}, z_{2}$ are independent samples of a zero-mean complex Gaussian random variable. The channel matrix $\mathbf{H}_{\mathcal{Q}}$ is a quaternion and we have $\mathbf{H}_{\mathcal{Q}} \mathbf{H}_{\mathcal{Q}}^{*}=\left(\left|h_{1}\right|^{2}+\left|h_{2}\right|^{2}\right) \mathbf{I}_{2}$. Two simple matched-filtering operations, $\mathbf{H}_{\mathcal{Q}}^{*} \mathbf{R}_{\mathcal{Q}}$ and $\mathbf{H}_{\mathcal{Q}}^{*} \mathbf{R}_{\tilde{\mathcal{Q}}}$ are performed to generate two candidate solutions, namely, $\hat{\mathbf{S}}_{\mathcal{Q}}$ and $\hat{\mathbf{S}}_{\tilde{Q}}$ which are then compared using the metric $\|\left[r_{1} r_{2}\right]-$ $\left[\begin{array}{ll}h_{1} & h_{2}\end{array}\right] \mathbf{S} \|^{2}$. The decoding of $b_{0}$ follows directly once the decision between $\mathbf{S}_{\mathcal{Q}}$ or $\mathbf{S}_{\tilde{\mathcal{Q}}}$ is made.

\section{Extension to 4 Transmit Antennas}

Consider the following example of a rate- 1 full-diversity complex quasi-orthogonal design [5], [7].

$$
\begin{aligned}
\mathcal{C} & =\left[\begin{array}{cccc}
x_{1} & x_{2} & y_{1} e^{j \theta} & y_{2} e^{j \theta} \\
-x_{2}^{*} & x_{1}^{*} & -y_{2}^{*} e^{-j \theta} & y_{1}^{*} e^{-j \theta} \\
-y_{1}^{*} e^{-j \theta} & -y_{2}^{*} e^{-j \theta} & x_{1}^{*} & x_{2}^{*} \\
y_{2} e^{j \theta} & -y_{1} e^{j \theta} & -x_{2} & x_{1}
\end{array}\right] \\
& \equiv\left[\begin{array}{cc}
\mathcal{Q}\left(x_{1}, x_{2}\right) & \mathcal{Q}\left(y_{1} e^{j \theta}, y_{2} e^{j \theta}\right) \\
-\overline{\mathcal{Q}}\left(y_{1} e^{j \theta}, y_{2} e^{j \theta}\right) & \overline{\mathcal{Q}}\left(x_{1}, x_{2}\right)
\end{array}\right]
\end{aligned}
$$

where the information symbols $x_{1}, x_{2}, y_{1}, y_{2}$ belong to MPSK constellation and the rotation angle $\phi$ is dependent on the signal constellation. For QPSK constellations (which are the focus of this letter), $\phi=\frac{\pi}{4}$ is the optimum rotation angle. Here, $\overline{\mathcal{Q}}$ denotes the complex conjugate (without transpose) of $\mathcal{Q}$.

We expand the signaling set and increase the rate to $\frac{9}{8}$ (for QPSK) by considering the following code

$$
\tilde{\mathcal{C}}=\left[\begin{array}{cc}
\tilde{\mathcal{Q}}\left(x_{1}, x_{2}\right) & \tilde{\mathcal{Q}}\left(y_{1} e^{j \theta}, y_{2} e^{j \theta}\right) \\
\overline{\tilde{\mathcal{Q}}}\left(y_{1} e^{j \theta}, y_{2} e^{j \theta}\right) & -\tilde{\tilde{\mathcal{Q}}}\left(x_{1}, x_{2}\right)
\end{array}\right]
$$

whose symbols are scaled by a factor $\frac{1}{K}$ as in the 2 TX case. For QPSK, encoding proceeds as follows. From each stream of nine information bits at the encoder, the first information bit selects between $\{\mathcal{C}\}$ and $\{\tilde{\mathcal{C}}$, whereas the last eight information bits are first mapped into 4 QPSK symbols $x_{1}, x_{2}, y_{1}, y_{2}$ and then space-time encoded as follows:

$$
\begin{aligned}
& \left(b_{0}=0\right) \Rightarrow\left[\begin{array}{cc}
\mathcal{Q}\left(x_{1}, x_{2}\right) & \mathcal{Q}\left(y_{1} e^{j \theta}, y_{2} e^{j \theta}\right) \\
-\overline{\mathcal{Q}}\left(y_{1} e^{j \theta}, y_{2} e^{j \theta}\right) & \overline{\mathcal{Q}}\left(x_{1}, x_{2}\right)
\end{array}\right], \\
& \left(b_{0}=1\right) \Rightarrow\left[\begin{array}{cc}
\tilde{\mathcal{Q}}\left(x_{1}, x_{2}\right) & \tilde{\mathcal{Q}}\left(y_{1} e^{j \theta}, y_{2} e^{j \theta}\right) \\
\overline{\tilde{\mathcal{Q}}}\left(y_{1} e^{j \theta}, y_{2} e^{j \theta}\right) & -\overline{\tilde{\mathcal{Q}}}\left(x_{1}, x_{2}\right)
\end{array}\right]
\end{aligned}
$$

Following the analysis for the 2 TX case in Section II-C, it is straightforward to show that to ensure full diversity, we must have $K \neq 1$.

\section{NUMERICAL RESUlTS}

We assume QPSK modulation, a single antenna at the receiver, and coherent ML decoding. Figure 2 shows that the ratio $\frac{C G}{P M P R}$ reaches its maximum at $K=\sqrt{3}$ which corroborates our analysis in Section II-C. In Figure 3, we compare our proposed rate $-\frac{5}{4}$ code with the Alamouti [1] code using the measure of Effective Throughput $\eta$ defined as $\eta=(1-F E R) * R * \log _{2}(M)$, where $\mathrm{R}$ is the code rate, $M$ is the constellation size, and $F E R$ denotes the frame error rate. This Figure shows that at high SNR (where FER $\approx 0$ ), 


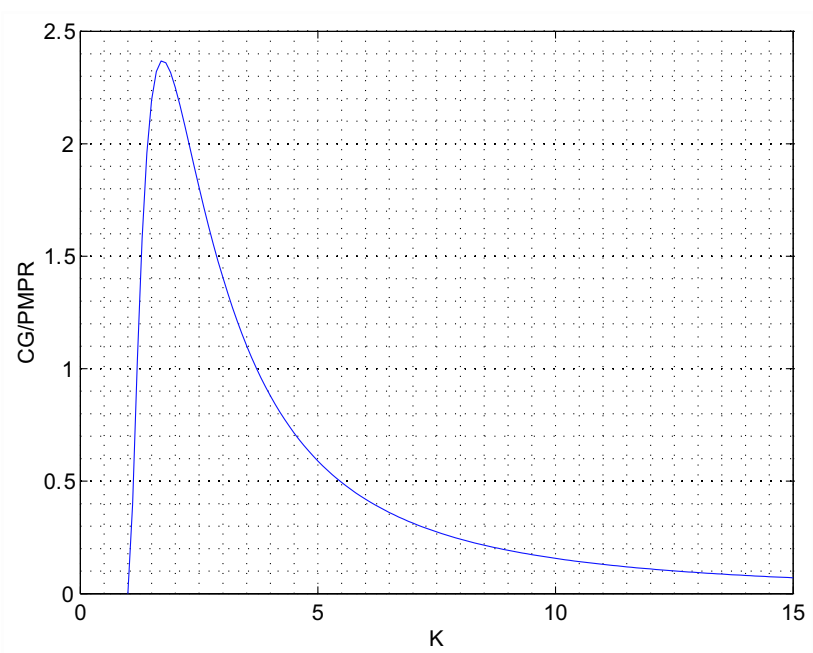

Fig. 2. CG to PMPR ratio for different $K$ values.

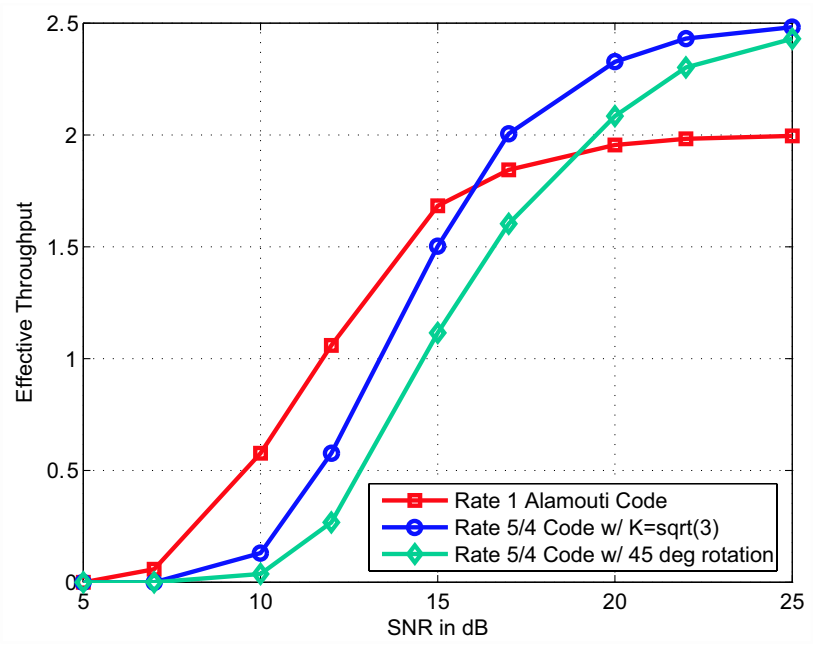

Fig. 3. Effective throughput comparison between Alamouti code and proposed rate- $\frac{5}{4}$ code for QPSK and 2 TX.

our code achieves a higher throughput level of 2.5 bits per channel use (PCU) whereas the achievable throughput for the Alamouti code is 2 bits PCU. We can observe a cross-over point at an input SNR level of $16 \mathrm{~dB}$. Since it is reasonable to assume that we know the operating input SNR level at the transmitter, we can switch between the Alamouti code and our proposed code to maximize throughput at all SNR levels. Figure 3 also depicts the achievable throughput of a rate- $\frac{5}{4}$ code that uses pure rotations (instead of power scaling) of the symbols in the main diagonal of codewords in $\tilde{\mathcal{Q}}$ by 45 degrees to ensure full-diversity and maximize coding gain. It demonstrates that optimum power scaling achieves higher throughput than optimum rotation for all values of SNR. Finally, Figure 4 shows that at high SNR, our proposed 4 TX code (with an optimized $K=\sqrt{2}$ ) achieves a throughput level of 2.25 bits PCU whereas the achievable throughput for the quasi-orthogonal design with 45 degree rotation [7] is 2 bits PCU and the SNR crossover point is at $14 \mathrm{~dB}$.

The power scaling factor $K$ ensures full-diversity and highrate at the cost of reduced coding gain; the cross-over point is a function of $K$. Different values of $K$ correspond to different

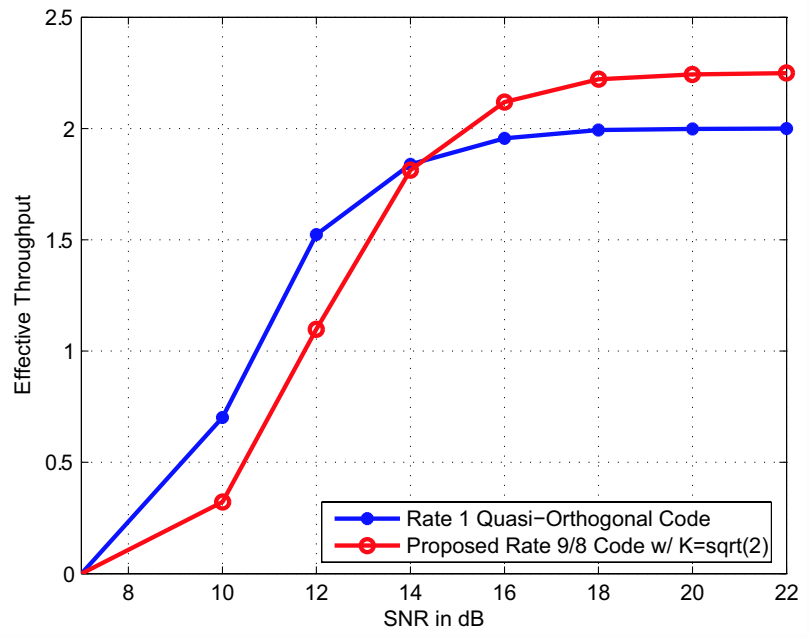

Fig. 4. Effective throughput comparison between rate-1 quasi-orthogonal code and proposed rate- $\frac{9}{8}$ code for QPSK and 4 Tx.

tradeoffs between control over PMPR and reduction in coding gain. It is also possible to turn this coding loss into gain by introducing block codes for the fading channel as in [6]. For an overall rate of 1 , this combination of coding techniques outperforms the Alamouti code [1] at the price of higher decoding complexity.

\section{CONClusion And Future WORKS}

We exploited the algebraic structure of quaternions to design and optimize a novel high-rate, full-diversity STBC for 2 transmit antennas. We introduced the concept of selective power scaling to guarantee full diversity for the designed code. The power scaling factor was further optimized to maximize available coding gain while minimizing transmit signal peakto-minimum power ratio. Furthermore, we extended this concept to 4 TX using the quasi-orthogonal STBC structure where we designed a new rate- $\frac{9}{8}$ code.

Currently, we are investigating the possibility of increasing the rate of our 2 TX STBC to accommodate 2 and 3 additional information bits (in stead of just 1 additional bit as in Section II-A) using additional members of the $\mathcal{Q}$ and $\tilde{\mathcal{Q}}$ families of orthogonal matrices [3].

\section{REFERENCES}

[1] S. M. Alamouti, "A simple transmit diversity technique for wireless communications," IEEE J. Select. Areas Commun., vol. 16, pp. 14511458, Oct. 1998.

[2] V. Tarokh, H. Jafarkhani, and A. R. Calderbank, "Space-time block codes from orthogonal designs," IEEE Trans. Inform. Theory, vol. 45, pp. 1456-1467, July 1999.

[3] H. Jafarkhani and N. Seshadri, "Super-orthogonal space-time trellis codes," IEEE Trans. Inform. Theory, vol. 49, pp. 937-950, Apr. 2003.

[4] V. Tarokh, N. Seshadri, and A. R. Calderbank, "Space-time codes for high data rate wireless communications: performance criterion and code construction," IEEE Trans. Inform. Theory, vol. 44, pp. 744-765, Mar. 1998.

[5] H. Jafarkhani, "A quasi-orthogonal space-time block code," IEEE Trans. Commun., vol. 49, pp. 1-4, Jan. 2001.

[6] N. Seshadri, and C. E. W.Sundberg, "Multilevel trellis coded modulations for the Rayleigh fading channel," IEEE Trans. Commun., vol. 41, pp. 1300-1310, Sept. 1993.

[7] W. Su and X.-G. Xia, "Signal constellations for quasi-orthogonal spacetime block codes with full diversity," IEEE Trans. Inform. Theory, vol. 50, pp. 2331-2347, Oct. 2004. 